\title{
Investigating the Neural Basis of Brain-Computer Interface (BCI)-based Stroke Rehabilitation
}

\author{
Timm Meyer, Jan Peters, Thorsten O. Zander, Doris Brötz, Surjo R. Soekadar, \\ Bernhard Schölkopf, Moritz Grosse-Wentrup
}

\begin{abstract}
The application of brain-computer interfaces (BCI) shows promising results in stroke rehabilitation, but the underlying neural substrates and processes of successful BCI-based neurorehabilitation remain unclear. The goal of our present work was to identify the brain areas associated with successful visuomotor integration and motor learning (VMIL), and investigate their connection with successful sensorimotorrhythm (SMR)-modulation commonly used in stroke rehabilitation related BCI systems. Our hypothesis was that neural processes associated with VMIL are linked to characteristics of the SMR, and thus share a common neural basis. Preliminary results indicate that the areas used to predict the current state of VMIL overlap with, but are not confined to, those areas used for SMR-based BCI training in stroke rehabilitation. This supports our hypothesis that VMIL and successful SMR modulation used in stroke BCI training share a common neural basis.
\end{abstract}

\section{INTRODUCTION}

Stroke is one of the leading causes of adult disabilities, with about $80 \%$ of patients being affected by motor impairment [1]. Even after six months of intense rehabilitation efforts about $50 \%$ of patients still show motor impairments [2]. In recent years, the application of brain-computer interfaces (BCI) in stroke rehabilitation has increased [3] [4] [5]. Most of these paradigms reward successful modulation of ipsilesional brain oscillatory activity, e.g. sensori-motor rhythms (SMR, 8-15Hz). However, it is unclear how such training of ipsilesional SMR modulation relates to motor recovery.

We present results of a pilot study, in which we combined an EEG-system with a seven degree-of-freedom (DoF) robot arm to study the neurophysiological correlates of visuomotor integration and learning (VMIL) during 3D reaching movements. The primary goal of this study is to identify brain regions, across subjects, that are associated with improved performance in a VMIL task. We find that the extent of SMR modulation, which is typically trained in BCIs, is linked to the state of VMIL. This indicates a common neural basis for BCI training and VMIL. Furthermore, we find regions/processes involved in VMIL that have not yet been considered for use in BCI-systems.

Timm Meyer, Jan Peters, Thorsten O. Zander, Bernhard Schölkopf and Moritz Grosse-Wentrup are with the Max Planck Institute for Intelligent Systems, Tübingen, Germany

Jan Peters is also with the Technische Universität Darmstadt, Intelligent Autonomous Systems Group, Darmstadt, Germany

Doris Brötz and Surjo R. Soekadar are with the Institute of Medical Psychology and Behavioural Neurobiology, MEG Center, University of Tübingen, Germany

This work was supported by the Bundesministerium für Bildung und Forschung (BMBF, Förderzeichen 01GQ0831) and the Deutsche Forschungsgemeinschaft (DFG)

\section{MATERIAL AND METHODS}

Six healthy subjects ( 3 male, 3 female; mean age $29.5 \pm$ 4.5 ), recruited from the local student body, participated in the present VMIL study. All subjects were right-handed and thus conducted the experiment with their right arm.

The subjects were attached with their arm to the robotic system (Figure 1) and performed four blocks of 50 trials, interleaved with a brief one minute intermission. The goal of each trial was to move the robot arm to reach a target visualized in 3D on a computer screen. This screen displayed the current position of the robotic arm's end-effector as a blue ball. Depending on the current phase, the target was either not shown (baseline (5 seconds)), displayed as a yellow ball (planning (2.5 - 4 seconds)), or displayed as a green ball (move). The initial position, in which the arm was hanging beside the body, was chosen independently by each subject. For each trial the target was randomly chosen from an area that could be reached comfortably by the subject. Due to the robotic arm's DoFs, subjects are able to perform a large variety of natural movements. While subjects moved the robotic arm, continuous visual feedback was provided about the end-effector's current position.

Throughout the experiment, a 120-channel EEG was recorded at $1 \mathrm{kHz}$ sampling rate, using active EEG electrodes and a QuickAmp amplifier (BrainProducts, Gilching, Germany). Electrodes were placed according to the 10-20 system, with $\mathrm{Cz}$ as the initial reference electrode. All data were re-referenced to common average reference offline.

To track each subject's learning process over the course of the experiment, we computed the normalized time-totarget (TTT) for each trial, i.e. the time required from the instruction to initiate the movement to reaching the target, divided by the distance. We observed a continuous decline in TTT over the course of the experiment, reflecting successful VMIL processes (Figure 2). This trend is captured by the low frequencies of the TTTs' power spectral density (PSD) (Figure 3).

In the following, we investigate whether TTT can be predicted on a trial-to-trial basis from EEG recorded in the upcoming target's planning phase. To do so, we highpass filtered the recorded EEG at $3 \mathrm{~Hz}$, and separated the data into (ideally) statistically independent components (ICs). This was done by first reducing the data to 64 principal components, then applying the SOBI-algorithm [6]. We inspected each IC manually and rejected those which were not of cortical origin. We then computed log-bandpower of 


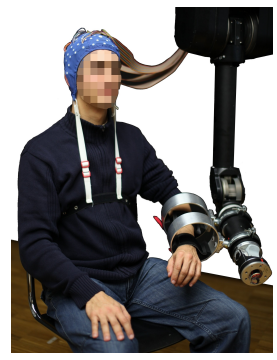

Fig. 1: Subject wearing an EEGcap while being attached to the seven DoF Barrett WAM arm.

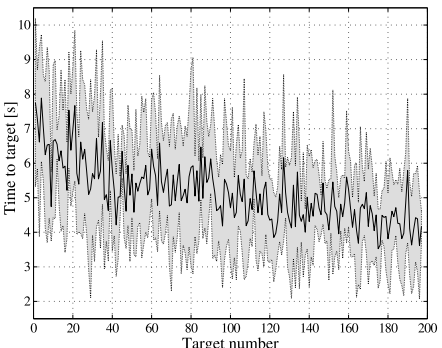

Fig. 2: Mean and standard deviation of the changes in time-to-target across the experimental session for the six subjects.

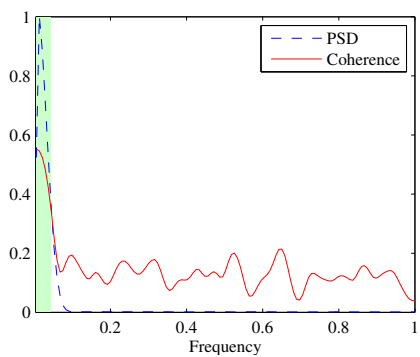

Fig. 3: Power spectral density of time-totarget values and coherence of prediction. Green shaded area shows significant frequencies. each non-artifactual IC in three frequency bands (using an FFT in conjunction with a Hanning window): $\mu(8-14 \mathrm{~Hz})$, low $\beta(20-30 \mathrm{~Hz})$, and $\gamma(55-85 \mathrm{~Hz})$. We used backward elimination feature selection [7], based on the correlation between predicted and real performance, in conjunction with 10-times cross validation, to fit a linear regression model that predicts TTT from the ICs' bandpowers. We then calculated the coherence between the predicted and the actual TTT and estimated significance with a permutation test. For this test, the trial order of the input variables was permuted, and coherence was calculated. This was repeated 5,000 times and, to correct for multiple comparisons, significance was tested using a false discovery rate of .01 [8].

\section{RESULTS}

The employed permutation test rejected the nullhypothesis that the temporal structure of the features does not provide any information on the current state of VMIL for all frequencies between [.0078 - .0465] Hz of the TTTs' PSD. This implies that our prediction captures the global learning trend, as this trend is represented by the low frequencies of the TTTs' PSD (Figure 3). In order to identify cortical areas relevant for TTT-prediction, the regression weights of the linear model were projected to the cortical level as described in [9] (Figure 4). While source localisation for the $\mu$ frequency band shows a clear focus covering parts of the contralateral somatosensory and motor cortex (Figure $4 a)$, it is less focused for the $\beta$ - and $\gamma$-band. In the $\beta$ band, parietal areas appear most relevant for performance prediction. In the $\gamma$-band, contralateral frontal cortex areas and the supplementary motor area show relevancy.

\section{DISCUSSION AND CONCLUSIONS}

The extent of the SMR's change during the course of one session correlates negatively with the TTT and thus positively with the state of VMIL (Figure 4a). This indicates that training to modulate the SMR, e.g. in BCI training, could enhance the capability of VMIL, which in turn might be beneficial for stroke rehabilitation. These results, especially regarding the $\mu$ frequency band, support the hypothesis that BCI training may facilitate VMIL and thus support rehabilitation processes. However, the $\beta$ and $\gamma$ frequency band indicate that BCI training could be improved by extending to areas and regions beyond those generating SMR

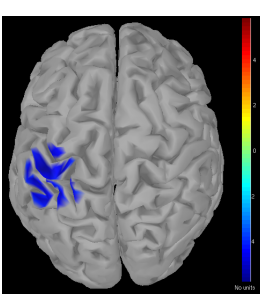

(a) $\mu(8-14 \mathrm{~Hz})$

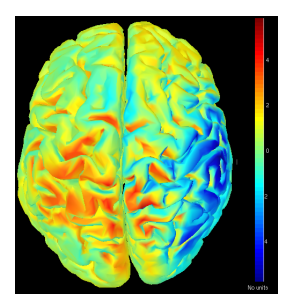

(b) $\beta(20-30 \mathrm{~Hz})$

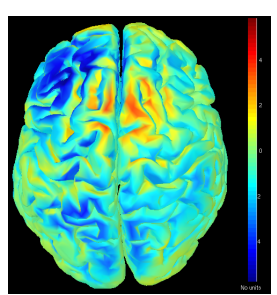

(c) $\gamma(55-85 \mathrm{~Hz})$
Fig. 4: Cortical areas relevant for TTT-prediction in the $\mu, \beta, \gamma$ range (with a cut-off at $70 \%$ in Figure $4 a$ )

Based on these results, we plan to conduct future studies, investigating whether the connection between successful VMIL and SMR modulation exists in stroke patients as well.

\section{REFERENCES}

[1] P. Langhorne, F. Coupar, and A. Pollock, "Motor recovery after stroke: a systematic review," The Lancet Neurology, vol. 8, no. 8, pp. 741-754, 2009.

[2] L. Jones, F. van Wijck, M. Grealy, and P. Rowe, "A changing stroke rehabilitation environment: Implications for upper limb interventions," in Pervasive Computing Technologies for Healthcare (PervasiveHealth), 2011 5th International Conference on. IEEE, 2011, pp. 374-378.

[3] M. Grosse-Wentrup, D. Mattia, and K. Oweiss, "Using brain-computer interfaces to induce neural plasticity and restore function," Journal of Neural Engineering, vol. 8, p. 025004, 2011.

[4] K. Chua, C. Guan, K. K. Ang, C. Kuah, K. S. Phua, C. Wang, H. Zhang, J. Lee, E. Arul, W. Low, B. T. Ang, and K.-H. Kong, "Brain-Computer Interface-based (BCI) based arm robotic rehabilitation for Stroke: A feasibility study and randomized controlled trial," BRAIN INJURY, vol. 26, no. 4-5, pp. 492-493, APR-MAY 2012.

[5] K. Ang, C. Guan, K. Chua, B. Ang, C. Kuah, C. Wang, K. Phua, Z. Chin, and H. Zhang, "Clinical study of neurorehabilitation in stroke using EEG-based motor imagery brain-computer interface with robotic feedback," in Engineering in Medicine and Biology Society (EMBC), 2010 Annual International Conference of the IEEE. IEEE, 2010, pp. 5549-5552.

[6] A. Belouchrani, K. Abed-Meraim, J.-F. Cardoso, and E. Moulines, "A blind source separation technique using second-order statistics," IEEE Transactions on Signal Processing, vol. 45, no. 2, pp. 434-444, 1997.

[7] R. Kohavi and G. John, "Wrappers for feature subset selection," Artificial intelligence, vol. 97, no. 1-2, pp. 273-324, 1997.

[8] Y. Benjamini and D. Yekutieli, "The control of the false discovery rate in multiple testing under dependency," Annals of statistics, pp. 11651188, 2001.

[9] M. Grosse-Wentrup and B. Schölkopf, "High gamma-power predicts performance in sensorimotor-rhythm braincomputer interfaces," Journal of Neural Engineering, vol. 9, no. 4, p. 046001, 2012. [Online]. Available: http://stacks.iop.org/1741-2552/9/i=4/a=046001 